\title{
PC-based Virtual Reality Surgical Simulation for Orthognathic Surgery
}

\author{
James Xia ${ }^{1,2 \& 3}$, Nabil Samman ${ }^{2}$, Chee Kai Chua ${ }^{1}$, Richie W.K. Yeung ${ }^{2}$, \\ Dongfeng Wang ${ }^{2}$, Steve Guofang Shen ${ }^{2}$, Horace H.S. Ip ${ }^{3}$, and Henk Tideman ${ }^{2}$ \\ ${ }^{1}$ Biomedical Engineering Research Center, School of Mechanical \& Production Engineering, \\ Nanyang Technological University, Singapore \\ j.xia@ieee.org \\ mckchua@ntu.edu.sg \\ ${ }^{2}$ Oral \& Maxillofacial Surgery, Prince Philip Dental Hospital, University of Hong Kong \\ j.xia@ieee.org \\ \{nsamman, htideman\}@hkucc.hku.hk \\ \{rwkyeung, dfwang, gfshen\}@hkusua.hku.hk \\ ${ }^{3}$ Department of Computer Science, City University of Hong Kong \\ j.xia@ieee.org \\ cship@cityu.edu.hk
}

\begin{abstract}
Complex maxillofacial deformations continue to present challenges in analysis, planning and correction beyond modern technology. The purpose of this paper is to present a virtual reality environment for surgeons to perform virtual orthognathic surgical planning in three dimensions. A surgical planning system, "PC-based virtual reality surgical simulation for orthognathic surgery" (VRSSOS), consists of four major stages: CT data post-processing and reconstruction; 3D color facial soft tissue model generation; virtual osteotomy planning; and, soft-tissue-change simulation. The surgical planning and simulation are based on $3 \mathrm{D} C \mathrm{CT}$ reconstructed bone model, whereas the soft tissue prediction is based on color texture-mapped and individualized facial soft tissue model. Our approach is able to provide a quantitative osteotomy-simulated bone model and prediction of post-operative appearance with photo-realistic quality. The predicted appearance can be visualized from any arbitrary viewing point using a low-cost PC-based system.
\end{abstract}

\section{Introduction}

Orthognathic surgery attempts to re-establish functional and aesthetic anatomy by repositioning displaced bones or by grafting and re-contouring the deformed bone contour. Soft tissue changes usually are accompanied along with these skeletal alterations. Despite of the advance in imaging modalities, the prediction of soft-tissuedeformation upon the bone-structure-change still poses major challenges to both surgeons and computer scientists. 
Three-dimensional (3D) studies in medicine started at the beginning of the 1970s. The cross-sectional imaging capability of computed tomography (CT) and three-dimensional reconstruction have led to a tremendous leap in diagnostic radiology. It can exactly record and represent the life size and shape of bone and soft tissue contour for precise bone surgery planning and simulation.

Surgical planning and simulation requires a conjunctive knowledge between medicine and computer graphics. Upon the points we presented above, the purpose of this study is to establish a virtual reality environment for orthognathic surgical planning, "PC-based virtual reality surgical planning and simulation for orthognathic surgery (VRSSOS)". It provides facilities for surgeons to make surgical plan, to simulate various orthognathic surgical procedures as they routinely performed in operating room and to predict soft tissue changes.

\section{VRSSOS Overview}

This virtual reality workbench consists of four major functions: CT data postprocessing and reconstruction; 3D color facial soft tissue model generation; virtual surgical planning and simulation; and soft-tissue-change prediction before operation (Fig.1). Surgical planning and osteotomy simulation were made on the reconstructed 3D bone structures, whereas soft tissue changes were then predicted on the individualized and color texture-mapped facial soft tissue model.

Our approach allows an immersive virtual reality surgical planning environment to be implemented on a relatively low-cost PC-based system when highend graphics workstations were normally required. Within the VRSSOS, a surgeon can get hold of a virtual "scalpel" to operate on a "virtual" patient, to execute various surgical plans, and to visualize the predicted post-operative results (Fig.2). The system outputs orthognathic surgical plans and a 3D color photo-realistic patient's facial soft tissue model. The osteotomy-simulated bone model and predicted color model can be rotated and visualized from any point of view.

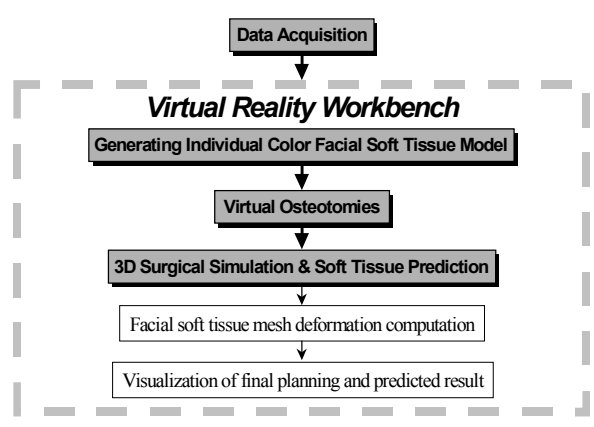

Fig. 1 System overview

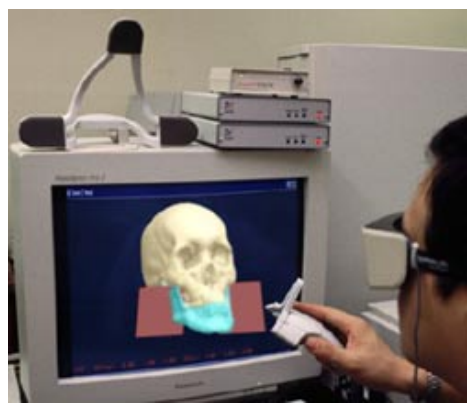

Fig. 2 Virtual “patient” on VRSSOS 
An IBM compatible Pentium $I I^{\circledR}-400$ personal computer (PC) was used. To create a virtual reality workbench, the virtual reality devices, such as Logitech $^{\circledR}$ threedimensional flying mouse system and CrystalEyes ${ }^{\circledR} 2$ eyewear, were also applied for this study.

The operating system was Microsoft ${ }^{\circledR}$ Windows $\mathrm{NT}^{\mathrm{TM}}$ Workstation 4.0 with Service Package 6 , whereas all the programs were developed by Microsoft ${ }^{\oplus}$ Visual $\mathrm{C}^{++T M}$ 6.0, OpenGL ${ }^{\circledast}$ and $\mathrm{VTK}^{\circledast}$.

\section{Clinical Data Acquisition}

Fourteen patients with maxillofacial deformities were randomly selected from a large pool of patients awaiting surgical correction of their deformity during December 1995 to December 1998. All patients were evaluated at the Surgical-Orthodontic Joint Assessment Clinic, Oral and Maxillofacial Surgery, University of Hong Kong.

Standard cephalometric radiographs were taken with fixed $110 \%$ magnification ratio. Diagnosis and preliminary operative plan were made by the cephalometric analysis and clinical examination.

The patient's Frankfort Plane was parallel to the horizontal plane during photography. Portraits were then scanned as 24 bit images into the computer via a Nikon $L S-20$ film scanner.

CT scanning was specially carried out for this study. All patients were examined by a $\mathrm{GE}^{\circledR}$ Pace CT Scanner at the Hong Kong Adventist Hospital, with a thickness of $2.0 \mathrm{~mm}$ and original $512 \times 512$ matrix with 16 bits. A rubber band was used to restrict the patient head's movement during CT scanning. The 2D axial slices started from the submandibular region and covered the whole head.

\section{Methods}

\subsection{CT Data Post-Processing and 3D Unique Coordinate System Creation}

CT data post-processing. For the true perspective of data visualization, image processing techniques were used to manipulate image contents to improve the results of subsequent processing and interpretation. The post-processing functions included: removing undesired objects, masking individual anatomical structure and the bone structure enhancement.

Segmentation was the process of classifying pixels in the image or volume. Depending on the scene complexity, it can be one of the most difficult tasks in the visualization process. Interactive segmentation was used if the threshold filter was not able to mask the bone in the CT dataset during the Marching Cubes process.

Three-dimensional reconstruction. A volumetric dataset was then prepared by using the Marching Cubes algorithm ${ }^{1,2}$ from the post-processed CT raw data. For fast 
rendering and shading, all the succeeding operations were based on this volumetric dataset.

Unique three-dimensional coordinate system. The visualization coordinate system

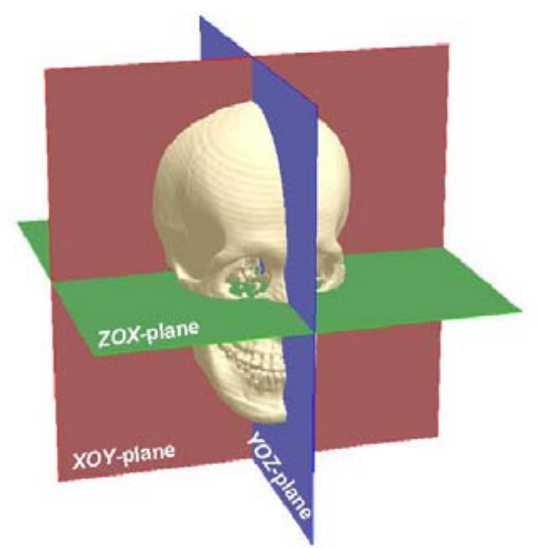

Fig. 3 3D coordinate system transformation represented what was visible to the observer. The $x, y$ coordinates specified location of object in a plane, whereas the $z$ coordinate represented the depth. Horizontal Plane was defined by right and left Porion, and the average coordinates of the right and left Orbitale. Frontal Plane was perpendicular to Horizontal Plane and through the right and left Porion. The Midsagittal Plane was set perpendicular to the Horizontal Plane and the Frontal Plane, and through the Nasion (Fig.3). ${ }^{3}$ After generating this unique coordinate system, 3D visualization and manipulation could be performed repeatedly, like cephalometric analysis.

\subsection{D Facial Model Generation}

Two techniques were applied in this stage: individualized facial model generation from a generic mesh and color texture mapping.

Generic facial soft tissue model. Our generic facial model is a triangular mesh consisted by 2665 vertices and 5302 triangles. ${ }^{3}$ This mesh was created from 3D bone and soft tissue which reconstructed from $\mathrm{CT}$ scans of a real human head. It contained the corresponding relationship of the coordinates between bone and soft tissue. Each coordinate on the bone surface had its own unique projection to the soft tissue mesh. When each point on the bone surface was changed, the corresponding coordinate of soft tissue would be changed accordingly. This allows us to simulate soft tissue deformation as a result of bone movement. Additionally, there were a series of builtin vertices representing facial outlines and features.

3D color facial model generation. The vertices controlling the facial features and outline were extracted from the $3 \mathrm{D}$ reconstructed soft tissues from CT scans, the generic mesh was then individualized as a texture coordinates by correspondence matching, interpolation and transformation computation based on those fiducial points.

The texture mapping was a procedure similar to pasting a picture to the surface of an object. Three digitized color portraits with the "third" dimension from reconstructed soft tissue were blended into a cartograph as a texture map.

After the cartograph was texture-mapped onto the 3D head model, a photorealistic model of human head was generated from frontal, right and left real color 
portraits. It could be rotated freely and visualized from any viewpoint at a real-time speed. All the procedures described in the texture-mapping stage were fully automatically, no interactive operation was required although it involved very complex computations.

\subsection{D Virtual Osteotomy Planning and Simulation}

This stage was to cut through the 3D reconstructed bone dataset with a surface and then to display interpolated data values on the surface, in order to achieve the virtual osteotomy simulation.

The bone surface was represented by millions of polygons and triangles. A fully automatic decimation computation was first applied on reconstructed bone surface to reduce the triangles up to $70 \%$, in order to keep the balance between maintaining bone structure details, obtaining an acceptable speed of rendering and reflash rate during cut-through the bone dataset. ${ }^{4}$

The data cutting operation required two pieces of information: a definition for the surface (cutting surface) and a dataset to cut (which bone to be cut). The virtual osteotomy was performed interactively and surgeon needed to decide which osteotomy would be used upon his clinical experience. ${ }^{5}$ Fig.2 showed the cutting planes of bilateral subsigmoid osteotomies were defined by moving a 3D mouse.

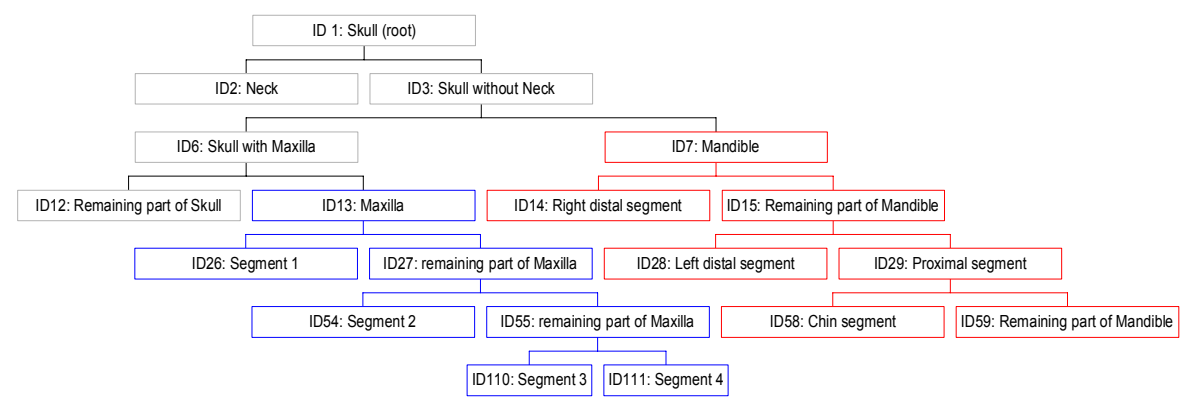

Fig. 4 Binary Tree Structure of simulated osteotomies

In the virtual osteotomy simulation, the Binary Tree Structure was used to identify the bone segments during maxillary and mandibular osteotomies ${ }^{5}$. Each bone segment was assigned a unique element identity (ID) automatically. A nonlinear structure, called a tree, consisted of nodes, branches and leaves. In the simulation of the operative procedures of a segmentalized Le Fort I osteotomy and bilateral vertical subsigmoid osteotomies with genioplasty, the root of the tree was the skull (ID1). Separating the neck from the skull, there were two nodes in the tree, the neck (ID2) and remaining part of the skull (ID3). Separating ID3 into ID6 and ID7, the maxilla (ID13) could be osteotomized from the skull without the mandible and the neck (ID6) - Le Fort I osteotomy. Four leaves with ID 26, 54, 110 and 111 were then assigned after the maxilla was segmentalized into four pieces. In the same principle, the mandible (ID7), also separated from ID3, was osteotomized as the right distal segment (ID14), the left distal segment (ID28) and the proximal segment (ID29) by 
bilateral subsigmoid osteotomies. The chin segment (ID58) was osteotomized from proximal segment (ID29) by genioplasty at last (Fig.4).

After osteotomies, each bone segment with

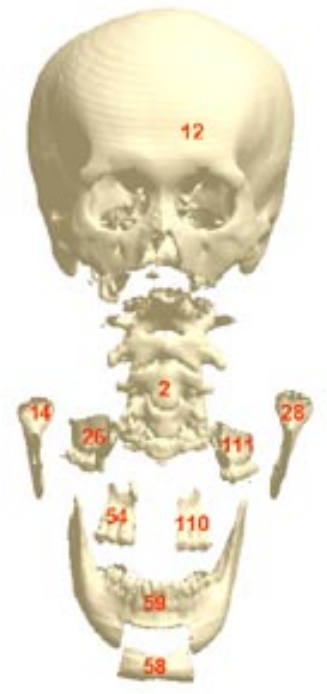

Fig. 5 osteotomized bone segments it's own ID could be manipulated separately in three dimensions, including translation, rotation, elevation, changing rotation center, displaying movement parameter, color masking, scaling, range boundaries and switching each object to visible or invisible (Fig.5).

\subsection{D Soft Tissue Simulation}

It is a soft tissue deformation scheme for simulating the soft-tissue-change according the underlay bonemovement during planning. Two soft tissue deformation algorithms were created for this scheme: the Surface Normal-based Model Deformation Algorithm and the Ray Projection-based Model Deformation Algorithm. ${ }^{6}$ The corresponding relationship of the vertices of triangles between the bone and the soft tissue surface was computed and saved as an intersection data file. For the ray projection-based model deformation, the Cylinder sampling model was applied to compute the intersection data along the rayprojection from origin of coordinate, between Eyebrow level and Labiomental Fold level. The Sphere sampling model was applied to calculate the remaining parts along the ray projection. If the Surface Normal of the vertex inferior to the plane of landmark Labiomental Fold was equal to or smaller than minus $10^{\circ}\left(\leq-10^{\circ}\right)$, the intersection data was computed along the direction of surface Normal instead of the ray projection for the surface normal-based model deformation.

To orchestrate the soft tissue prediction with different operation types, the parameters of bone and soft tissue movement ratios were transferred from intervened ratio files correspondingly. ${ }^{7}$

Three-dimensional color facial texture-mapping technique was applied again to regenerate a color photorealistic facial model after soft tissue deformation.

The soft tissue changes were simply predicted by interactively and intraoperatively dragging the

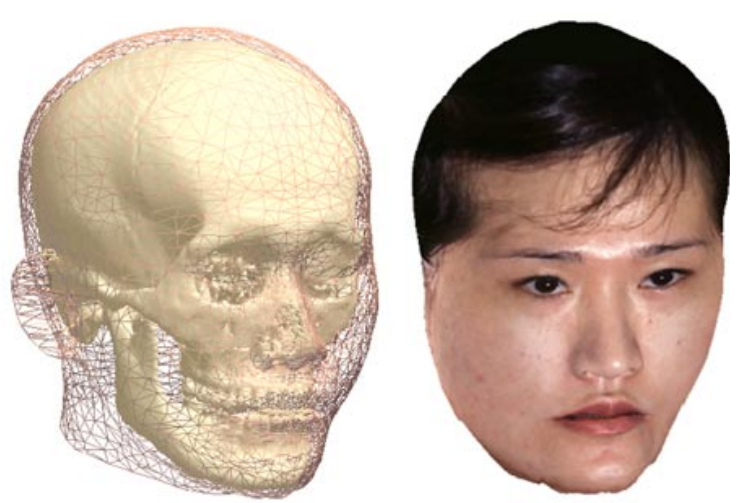

Fig.6 Soft tissue deformation after virtual osteotomies bone segments to desired position. When the surgeon translated, rotated and elevated each bone segment, soft tissue model was correspondingly deformed and texture was 
re-mapped from the cartograph in a real-time speed (Fig.6). A predicted threedimensional facial visualization with color photo-realistic quality was precisely created. Both the bone and soft tissue models could be rotated freely and visualized immediately from arbitrary observing angle with zoom-out or zoom-in. The each object of the bone and soft tissue also could be masked as visible or invisible in order to get the best visualization during the virtual operation.

\section{Clinical Trail}

To date, 14 cases have been planned using integrated two- and three-dimensional data and analysis. Surgical planning and simulation has been performed by this VRSSOS system. The post-operative visualization is predicted before operation.

The surgeon can use different combinations of short-cut keys, twodimensional mouse, three-dimensional flying mouse and CrystalEye eyewear, to perform the virtual osteotomies, to move bone segments and simulate soft tissue changes (Fig.2). The operator's clinical experience is critical for achieving a successful planning.

Based on the procedures and time requirements for analysis, six stages of treatment planning are available. A typical case with mandibular hyperplasia and mild paranasal deficiency was presented. The operative procedures were: Le Fort I osteotomy with four pieces segmentation, bilateral subsigmoid osteotomies and genioplasty.

The first stage involves data acquisition and clinical evaluation, such as CT scanning, color portraits capturing, cephalometric analysis and primary clinical assessment. This requires 2 to 7 days of effort.

The second stage involves $\mathrm{CT}$ raw data post-processing, including removing undesired objects, enhancement processing, masking bone structure, etc. This requires 2 to 3 hours of effort.

The third stage involves interactive visualization of the three-dimensional images, including the volumetric dataset generation, 3D coordinates geometry transformation, etc. This requires about 1 hour of effort (Fig.3).

The fourth stage of planning is to generate individual facial soft tissue model with a color texture mapping. This procedure requires about 1-2 hours.

The fifth stage of planning is to make Decimation computation and to perform varieties of procedures of virtual "osteotomies" in virtual reality environment. This step is only operated on the bone. It requires about 1 hour or less (Fig.5).

The sixth stage of planning is much quicker and requires about 10 to 15 minutes. This step is totally operated in the virtual reality environment with VR devices. The surgeon can move and rotate the osteotomized bone segments freely and observe the predicted result with full color visualization. The operator may also compare results between the predicted and the original facial models in threedimensional visualization (Fig.6).

A planning and simulation session was held at the computer system with the maxillofacial team for review and final modifications. The quantitative translation and rotation data of each object could be displayed respectively. The final result of 
planning and simulation can be visualized from arbitrary viewpoints in the virtual reality workbench.

Follow-up was made six months after operation. A comparison, between original color portraits, predicted facial model, postoperative portraits, simulated osteotomies and bone structures after surgery, is shown in Fig.7. Fig. 8 shows another case with the same operative procedures.
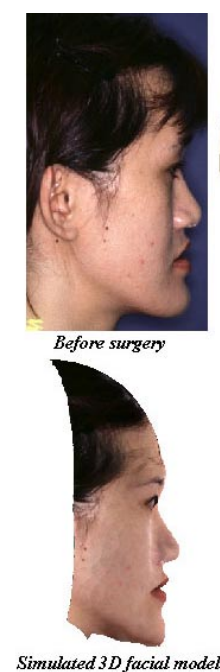

Simulated $3 D$ facial model
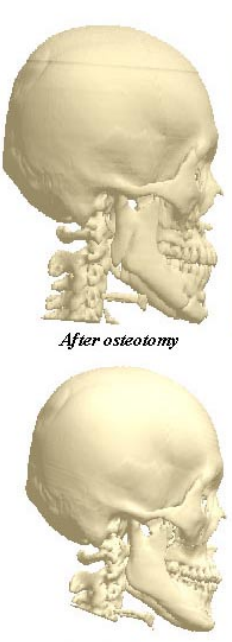

Simulated osteotomy

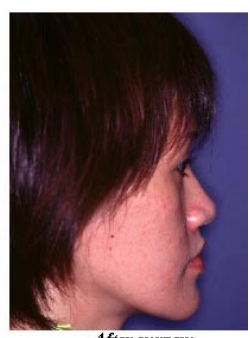

After surgery

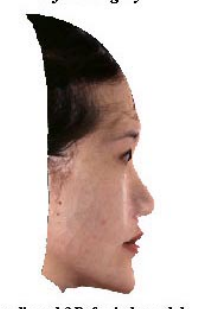

Predicted 3D facial model

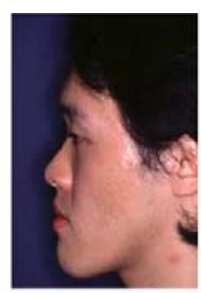

Before surgery

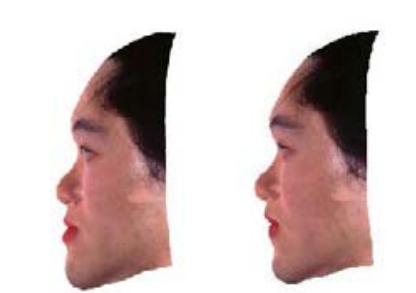

Simulated 3D facial model Predicted 3D facial model

Fig. 7 Comparison between Pre-OP and Post-OP

\section{Discussion}

Surgical planning and simulation has wide applications, such as for orthognathic surgery, craniofacial surgery, cleft palate, trauma, distraction osteogenesis, etc. In general, surgical simulation is to perform the "surgery" in the computer and to construct a "fake" image of patient's post-surgical appearance before operation.

Three-dimensional imaging technology is now widely available. It is used to aid in the comprehension and application of volumetric data to diagnosis, planning and therapy. ${ }^{8}$ There are several studies on surgical planning for maxillofacial and craniofacial surgery. Altobelli et al used the three-dimensional surface reconstruction from CT data to plan craniofacial operations. ${ }^{9}$ Cephalometric analysis was integrated with 3D CT reconstruction to quantify the skeletal deformities and to assist in the design of the surgical procedure. Interactive techniques were developed to simulate osteotomies and skeletal movement in three dimensions on the computer-generated surface images. It took about one or two days for bone surgery simulation on an extremely powerful high-end graphic workstation. Three-dimensional soft tissue prediction based on bone movement was not included. 
Stereolithographic biomodeling is a modern technology that allows 3D CT data to be used to manufacture solid plastic replicas of anatomical structures (biomodels). ${ }^{10}$ It can produce the life-sized deformed bone structures, so that surgeon can directly observe patient's deformity and make surgical planning on the biomodel before operation. It is good for planning complex craniofacial surgery, however, it needs very expensive Stereolithography Apparatus (SLA) machine to fabricate this biomodel, and soft-tissue-change prediction is still not included.

Soft-tissue-change prediction is an important aspect in surgical planning, especially in orthognathic surgery. The orthognathic surgery is not only to correct the mal-functions and to re-locate mal-positioned bones, but also for aesthetic purpose. Currently soft tissue simulation are still mainly focused on the two-dimensional profile prediction (color video imaging techniques) combining with cephalometric analysis in recent two decades, ${ }^{11,12}$ only a few studies involved in three-dimensional

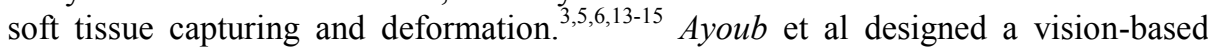
three-dimensional facial data capture system (photogrammetry) for the planning of maxillofacial operations. ${ }^{13,14}$ However, the relationship between soft tissue and bone is still remaining a question.

From the methods discussed above, there is no VR-based 3D system to make a precise orthognathic surgical planning and soft tissue prediction in the personal computer. The aim of this project is to combine three-dimensional visualization, virtual reality, color video imaging technique, surgical planning and simulation together, in order to solve the bone-soft tissue relationship problem and provide surgeon an ideal virtual reality workbench for orthognathic surgical planning, simulation and soft tissue prediction in a personal computer system. The workbench is designed not only for surgical planning, but for teaching purpose as well. This study has achieved the following general objectives:

- To create a three-dimensional virtual reality workbench for surgical planning, simulation and soft tissue prediction for orthognathic surgery;

- To generate fast 3D visualization, so that the multiple virtual osteotomies can be real-time performed;

- To obtain a final product — the quantitative osteotomy-simulated 3D bone model and the precise soft-tissue-change predicted 3D facial model with color photorealistic quality, which can be real-time visualized in three-dimensional virtual reality environment;

- All procedures can be operated in a conventional personal computer system with relatively low-cost VR devices, instead of extremely expensive high-end VR graphics workstation.

Considering the cost and radiation of whole skull CT scanning to the patient, this system is more likely to suitable for the cases with complex maxillofacial deformity, such as asymmetric deformity, cleft palate, distraction osteogenesis, trauma, where radiation and cost of CT scan need to be justified.

Future work will focus on a comparative study between predicted and postoperative models by surface anthropometry in order to verify the accuracy of the system quantitatively. 


\section{Acknowledgement:}

Authors thank Dr. Ron Kikinis, Associate Professor of Radiology and Director of Surgical Planning Laboratory, Brigham and Women's Hospital, Harvard Medical School, for his valuable suggestions and comments on this computer system.

\section{References}

1. Cline H.E., Lorensen W.E., Ludke S., et al: Two algorithms for the three-dimensional reconstruction of tomograms. Med Phys. 15: 320-327; 1988

2. Lorensen W.E. and Cline H.E.: Marching cubes: a high resolution 3D surface construction algorithm. Comput Graphics. 21: 163-169; 1987

3. Xia J., Wang D., Samman N., et al: Computer-assisted three-dimensional surgical planning and simulation - 3D color facial model generation. Int J Oral Maxillofac Surg. 29: 2-10, 2000

4. Schroeder W., Zarge J. and Lorensen W.: Decimation of triangle meshes. Comput Graphics. 26: 65-70, 1992

5. Xia J., Ip H.H.S., Samman N., et al: Computer-assisted three-dimensional surgical planning and simulation - 3D virtual osteotomy. Int J Oral Maxillofac Surg. 29: 11-7, 2000

6. Xia J., Samman N., Yeung RWK, et al: Computer-assisted three-dimensional surgical planning and simulation - 3D soft tissue planning and prediction. Accepted by Int J Oral Maxillofac Surg, 2000

7. Bell W.H.: Modern practice in orthognathic and reconstructive surgery. Saunders: Philadelphia, USA. Pp2186-96, 1992

8. Vannier M.W. and Marsh J.L.: Three-dimensional imaging, surgical planning, and imageguided therapy. Radiol Clin North Am. 34: 545-63, 1996

9. Altobelli D.E., Kikinis R., Mulliken J.B., et al: Computer-Assisted Three-dimensional planning in craniofacial surgery. Plast Reconstr Surg. 92: 576-85, 1993

10. D’Urso P.S., Barker T.M., Earwaker W.J., et al: Stereolithographic biomodelling in cranio-maxillofacial surgery: a prospective trial. J Craniomaxillofac Surg. 27: 30-37, 1999

11. Schultes G., Gaggl A. and Karcher H.: Accuracy of cephalometric and video imaging program Dentofacial Planner Plus in orthognathic surgical planning. Comput Aided Surg. 3: 108-14, 1998

12. Xia J., Wang D., Qi F., et al: Computer aided simulation system of orthognathic surgery. In Proceedings of Eighth IEEE Symposium on Computer-based Medical Systems, Lubbock, TX, USA: June 9-10, 1995. IEEE Computer Society Press: Los Alamitos, USA. Pp. 237-44, 1995

13. Ayoub A.F., Wray D., Moos K.F., et al: Three-dimensional modeling for modern diagnosis and planning in maxillofacial surgery. Int J Adult Orthodon Orthognath Surg. 11: 225-33, 1996

14. Ayoub A.F., Siebert P., Moos K.F., et al: A vision-based three-dimensional capture system for maxillofacial assessment and surgical planning. Br J Oral Maxillofac Surg. 36: 353-7, 1998

15. Keeve E., Girod S., Kikinis R., et al: Deformable modeling of facial tissue for craniofacial surgery simulation. Comput Aided Surg. 3: 228-38, 1998 